\title{
Development of highly porous activated carbon from Jacaranda mimosifolia seed pods for remarkable removal of aqueous-phase ketoprofen
}

doi: https://doi.org/10.1016/j.jece.2021.105676

url: https://www.sciencedirect.com/science/article/abs/pii/S2213343721006539

Authors: Jordana Georgin, Yamil L. de O. Salomón, Dison S.P. Franco, Matias S. Netto, Daniel G.A. Piccilli, Daniele Perondi, Luis F.O. Silva, Edson L. Foletto, Guilherme L. Dotto

\begin{abstract}
In this work, a high porous activated carbon from Jacaranda mimosifolia was developed and employed for ketoprofen adsorption. After the pyrolysis process at $973.15 \mathrm{~K}$, the material presented cavities with different sizes allocated on the particle surface. The material presented a $\mathrm{pH}$ at the point of zero charge of 4.1 with the best adsorption at $\mathrm{pH} 2$. The best adsorbent dosage was $0.72 \mathrm{~g} \mathrm{~L}-1$, corresponding to a removal of $96 \%$. The system reached the adsorption equilibrium after $120 \mathrm{~min}$ and was described by the linear driving force model. The isotherms revealed that the adsorption capacity decreased with the temperature and followed the Langmuir model, with a maximum adsorption capacity of $303.9 \mathrm{mg}$ g-1. This high capacity can be associated with the high surface area (928 m2 g-1) and pore volume $(0.521 \mathrm{~cm} 3 \mathrm{~g}-1)$ values. The thermodynamic values indicated that the adsorption system is spontaneous and exothermic. The enthalpy value indicates that the interactions between the adsorbent and adsorbate are physical. Regeneration tests showed a decreasing percentage of removal of $7.86 \%$ after 5 cycles. Finally, the adsorbent showed efficiency when treating a simulated effluent containing drugs and inorganic salts, showing the removal of $71.43 \%$.
\end{abstract}

\section{Keywords}

Activated carbon, Jacaranda mimosifolia, Ketoprofen, Adsorption 